\title{
A Unified Model for Current-Programmed Converters
}

\author{
F. Dong Tan, Member, IEEE, and R. D. Middlebrook, Life Fellow, IEEE
}

\begin{abstract}
A unified model is established for a currentprogrammed converter, which is both a modification and an extension of familiar models. Inclusion of the sampling effect allows the presence of an additional pole $\omega_{p}$ in the current-loop gain to be derived. The resulting final double-slope asymptote is fixed in position, and the crossover frequency cannot exceed half the switching frequency. A stability parameter, $Q_{s}$, determines the additional pole and describes the degree of peaking in the closed-loop transfer function. Experimental verification employs an analog signal injection technique.
\end{abstract}

\section{INTRODUCTION}

$\mathbf{C}$ URRENT programming has become the regulating scheme of choice in dc-to-dc converters owing to its advantages over duty-ratio programming such as better linenoise rejection, automatic overload protection, easy paralleling of multiple converters, and especially design flexibility in improving small-signal dynamics.

Since its initial conception [1], [2], a large number of smallsignal models have been proposed, for example, [3]-[12]. A brief nonexhaustive review of some of the previous efforts is presented in the following to show the evolution of models.

A low-frequency circuit-oriented approach was proposed in [5]. A modulator model (also referred to as "duty-ratio control law" by other authors) is derived by perturbation of an expression for the average inductor current in steady state. Then, the modulator model is interfaced with the state-space averaged model for the power stage to obtain a complete model for the entire converter system. Current-loop gains are identified and then absorbed. This approach has gained wide acceptance owing to its simplicity and the insight gained into the properties of current programming.

One of the major insights gained in [5] is that the crossover frequency of the current loop can in general be considered wideband, implying possible degradation of performance of a low-frequency model. To cope with potential deficiencies, a separate earlier work [4] needed to be used. This model is capable of predicting the well-known subharmonic oscillation which occurs, for example, when the duty ratio is greater than 0.5 and no compensating ramp is used. A general expression for the sampled-data version of the current-loop gain is derived, from which it is seen that the crossover frequency of the current loop is limited to approximately one third of the

Manuscript received March 18, 1994; revised March 13, 1995. F. Dong Tan is with QSC Audio Products, Costa Mesa, CA 92626 USA.

R. D. Middlebrook is with the Power Electronics Group, California Institute of Technology, Pasadena, CA 91125 USA.

IEEE Log Number 9412189 switching frequency. It will be shown later analytically as well as experimentally that this estimate is too conservative.

Another earlier work [12] suggested that the modulator gain factor $F_{m}$ can approach infinity at the limit of stability. A few authors have contested this result on the ground that Shannon's sampling theorem limits the crossover frequency to half the switching frequency. This apparent contradiction is resolved by the unified model. The unified model inherits the value of the gain factor but reveals further that the crossover frequency of the current-loop gain can not exceed half the switching frequency, not by limiting the value of the modulator gain factor, but by the presence of an additional pole due to sampling.

The modulator model in [5] was derived by perturbation of an expression for inductor average current in steady state. This procedure was disputed in [6], where it is argued that the smallsignal modulator model needs to be derived by perturbing an expression for inductor average current in perturbed state. Experimental measurements, however, did not support its prediction for the case where no compensating ramp is used. An explanation to this discrepancy is found in [7], which provides a geometric interpretation of the unified modulator gain to be proposed in this paper.

A continuous-time model was proposed in [8], where a modified Padé approximation of the complex exponential, accurate up to half the switching frequency, is used to approximate the sampled-data version of the current-loop gain derived in [4], allowing interpretation of sampled-data results in the continuous-time domain. Consequently, the mechanism behind the peaking, occurring at half the switching frequency in various closed-loop transfer functions, is seen to be due to the presence of a double right-half-plane zero at half the switching frequency. The expression for the current-loop gain crossover frequency, however, is found to be the same as in [4], which lacks experimental support and is inconsistent with the proposed corresponding closed-loop control-to-inductor-current transfer function. This inconsistency lies in the fact that difficulties were met in many attempts to derive the peaking at half the switching frequency, using the expression for the crossover frequency given in [4], [8]. Possible peaking at half the switching frequency in closed-loop transfer functions was confirmed in [4], [8], [9] as well as by the present authors in [21].

A model for switches in a current-programmed converter was proposed in [10], which enjoys easy implementation in computer simulation applications. The current-loop gain is not identified. A Q-factor is found to determine the degree of peaking in closed-loop transfer functions. 
In the following sections, a unified model for currentprogrammed converters is developed, which represents a lowfrequency modification and a high-frequency extension over previous models. A low-frequency modification leads to a unified modulator model, which results in improved predictions for several essential quantities of current-loop gain. An extension to include sampling effect allows the presence of an additional pole $\omega_{p}$ in the current loop to be derived. Two parameters are found to be central quantities of interest. Parameter $D_{\text {min }}^{\prime}$, the minimum value of $D^{\prime}$ to maintain stability of the current loop, shows explicitly the stabilizing effect of the compensating ramp. A "stability parameter" $Q_{s}$, related to the converter duty ratio and the compensating ramp, emerges as a central quantity of interest. Experimental verification employs an analog signal injection technique.

Notation conventions adopted here are as follows. Capital letters are used to indicate quantities associated with steadystate, hatted letters are quantities associated with small-signal perturbations, and small letters represent quantities associated with perturbed state, i.e., quantities which are the sum of capital and hatted letters.

\section{A UNIFIED MODUlator MODEL}

A brief introduction to the basics of current programming is first presented. Key steps of modeling a current-programmed converter are outlined. Then, a unified modulator model is derived.

\section{A. Basic Concept of Current Programming}

Figure 1(a) shows an illustrative diagram for a conventional duty-ratio-programmed buck converter. Because of the nature of the comparator (duty-ratio modulator), the duty ratio is determined when a ramp reaches a certain peak value set by a control voltage $v_{c}$. In common implementations, the ramp starts and ends with zero values and with a constant rising slope. Since the ramp is independent of any power stage variables, the duty ratio can be considered to be uniquely determined by the value of the control voltage $v_{c}$.

Figure $1(b)$ is an illustrative diagram for a currentprogrammed buck converter, where inductor current is sensed and compared to a control current $i_{c}$. A clock signal initiates each switching cycle at a constant frequency. Duty ratio $d$ is determined when inductor current $i_{l}$ reaches a certain peak value set by $i_{c}$. Thus, the duty ratio depends not only on the value of control current (which sets the peak value), but also on how long it takes the inductor current to reach the peak value. Also, this time duration is determined by the starting value and the up-slope of inductor current.

Use of inductor current to determine duty ratio introduces feedback into the converter. Thus, small-signal dynamics is expected to change from a typical double pole high Q-factor low-pass filter to an effective single dominant pole [5].

A model for a current-programmed converter consists of two parts: 1) the model for the power stage, and 2) the model for the modulator, or interchangeably, the duty-ratio control law. Since the state-space averaged model [11] is frequently adopted as the model of choice for the power stage, the actual
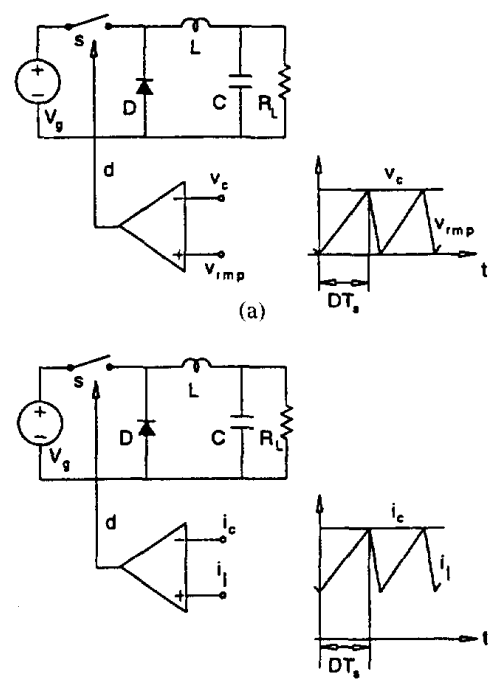

(b)

Fig. 1. (a) A duty-ratio-programmed buck converter, and (b) a current-programmed buck converter where the ramp function is obtained by sensing the inductor current.

task is reduced to the establishment of a modulator model in a form consistent with the power stage model.

A modulator model is expected to be expressed in terms of the average inductor current $i_{l}$, the average control current $i_{c}$, and an effective voltage $v_{o f f}$, which determines the slopes of the inductor current (to be derived later). Therefore, a desired form of small-signal low-frequency model of a currentprogrammed converter can be drawn as shown in Fig. 2.

This is essentially the same as in Figs. (11)-(13) of [5], except that a general canonical model for the power stage has been incorporated. Values of the parameters of the canonical model are well known and can be found in [11]. The modulator gain $F_{m}$ and coefficients $\alpha k$ (replacing $G_{6}$ ) and $\beta k$ (replacing $H_{3}$ ) reflect, respectively, the influence that the error current $\hat{i}_{c}-\hat{i}_{l}$, line voltage $\hat{v}_{g}$, and output voltage $\hat{v}$ may have on the duty ratio $\hat{d}$. The indicated frequency dependence for $F_{m}$ is not due to the modulator; it will be shown later that its physical origin is the sampling effect.

What remains to be done is to determine values of various gain factors in Fig. 2.

Figure 3 shows the definition of $v_{o f f}$ and $i_{o n}$ as originally proposed in [14]. Voltage $v_{o f f}$ is the sum of the voltages across the transistor $v_{t}$ and that across the diode $v_{d}$. The subcircuit in Fig. 3 is actually the underlying topological structure called the PWM tree in all PWM converters [14], [15], which guarantees the desired PWM switching in a dc-to-dc converter. Use of $v_{o f f}$ and $i_{o n}$ has the potential of unifying expressions for variables in different PWM converters, which will be evident in the derivation for the modulator model.

One of the useful results associated with $v_{\text {off }}$ is the following identity established in [13], [14], $V_{\text {of } f} / I_{o n}=R / M(D)$. This identity reveals that the dc operating parameter $R$, defined as the ratio of output dc voltage over the dc output current, can also be interpreted as a transfer resistance which has the 


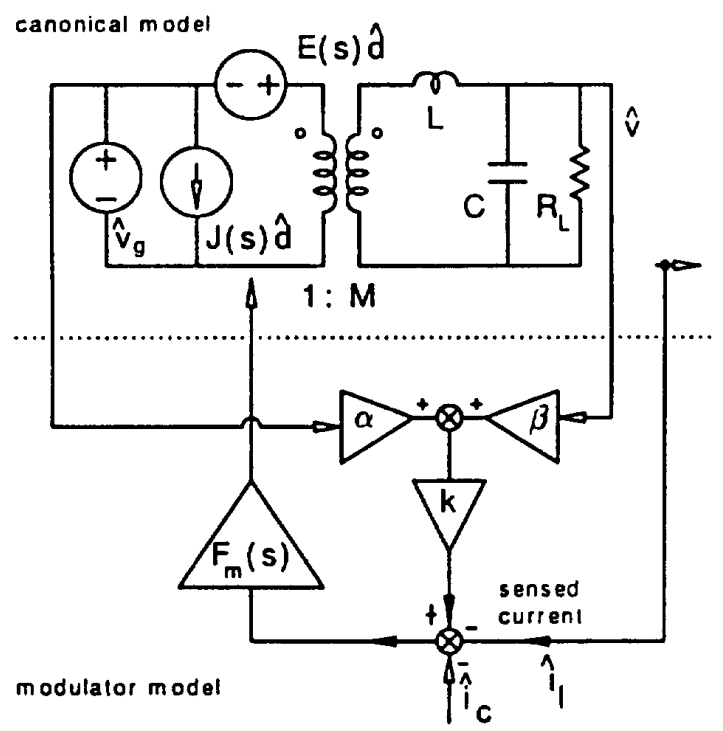

Fig. 2. The small-signal low-frequency model of a current-programmed converter consists of two parts: a model for the power converter, and a model for the duty-ratio modulator. It is shown later that the frequency response of the modulator gain $F_{m}(s)$ is a simple pole at $\omega_{p}=\left(\omega_{s} / 2\right) / Q_{s}$.

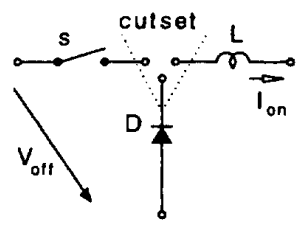

(a)

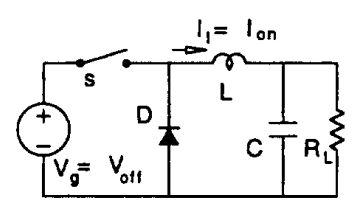

(b)
Fig. 3. Definition of (a) $v_{o f f}$, and (b) $i_{o n}$ in the PWM tree structure. Voltage $v_{o f f}$ is the sum of voltages across, and current $i_{o n}$ is the sum of currents through, the two switching devices.

TABLE I

Simplified EXPRESSIONS FOR QUaNTITIES IN THE CANONICAL Circuit Model for Three Basic ConverTers

\begin{tabular}{|c|c|c|c|}
\hline & $M(D)$ & $E(s)$ & $J$ \\
\hline \hline Buck & $D$ & $\frac{V_{\text {off }}}{D}$ & $I_{\text {on }}$ \\
\hline Boost & $\frac{1}{D^{\prime}}$ & $V_{\text {off }}\left(1-\frac{s L}{D^{\prime} V_{\text {off }} / I_{\text {on }}}\right)$ & $\frac{I_{\text {on }}}{D^{\prime}}$ \\
\hline Buck/Boost & $\frac{D}{D^{\prime}}$ & $V_{\text {off }}\left(1-\frac{s L}{D^{\prime} V_{\text {off }} / I_{\text {on }}}\right)$ & $\frac{I_{\text {on }}}{D^{\prime}}$ \\
\hline
\end{tabular}

value of $V_{o f f} / I_{o n}$ scaled by $M(D)$. Table I presents modified expressions for the quantities in the canonical model [11]. The unifying power of $v_{o f f}$ and $i_{o n}$ is evident.

Characterization of a converter is frequently pursued in terms of terminal quantities such as, for example, input voltage and output voltage. Under this circumstance, use of $v_{o f f}$ is not always convenient since $v_{o f f}$ is defined in terms of $v_{t}$ and $v_{d}$, which are not necessarily terminal quantities. To remedy this,

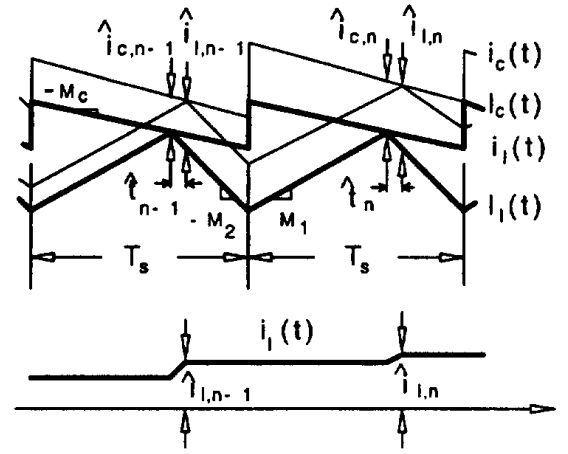

Fig. 4. Geometries of inductor currents in steady (in thick lines) and perturbed (in thin lines) states. Essential information about the modulator model can be obtained from the geometries.

a relation is derived which connects $\hat{v}_{o f f}$ directly to input voltage $\hat{v}_{g}$, and output voltage $\hat{v}$,

$$
\hat{v}_{o f f}=\alpha \hat{v}_{g}+\beta \hat{v}
$$

where $\alpha, \beta \in\{0,1\}$ (details in [23]).

\section{B. A Unified Modulator Model}

Figure 4 shows inductor current waveforms in steady state (in solid lines) as well as in perturbed state (in dashed lines), where $-m_{c}$ is the slope of the compensating ramp, $m_{1}$ is the up-slope and $-m_{2}$ is the down-slope of the inductor current.

The geometries of the waveforms provide fundamental information for derivation of a modulator model. From the geometry of the inductor current waveform in steady state (in solid lines) and with slopes $m_{1}, m_{2}$, and $m_{c}$ constant, one can write two equations:

$$
\left\{\begin{array}{l}
i_{l}=i_{c}-m_{c} d T_{s}-\frac{1}{2} m_{1} d T_{s} \\
i_{l}=i_{c}-m_{c} d T_{s}-\frac{1}{2} m_{2} d^{\prime} T_{s} .
\end{array}\right.
$$

Equation (2) is a set of simultaneous equations. They have to be met simultaneously. Many previous authors overlooked this point, which led to incomplete models. More importantly, conventional approaches can not be simply applied. Elimination of variables is not useful, since all the variables need to be kept in order to obtain a complete characterization. Linear combination of two equations cannot be used either, since there are infinite possible combinations which yield the same average value of inductor current. This uncertainty is actually a manifestation of the fact that the average value of inductor current is determined by the instantaneous value of inductor current at the time instant $t=D T_{s}$, when $i_{l}$ reaches the peak value set by $i_{c}$, not the other way around. In fact, (2) can also be considered as the instantaneous value of the perturbed averaged current at the time instant.

To unify the two equations in (2) into a single expression, one can use the following general expressions, originated in [13], for $m_{1}$ and $m_{2}$ :

$$
\left\{\begin{array}{l}
m_{1}=d^{\prime} v_{o f f} / L \\
m_{2}=d v_{o f f} / L
\end{array}\right.
$$


Substitution of (3) into either expression in (2) leads to the same expression:

$$
i_{l}=i_{c}-m_{\mathrm{c}} T_{s} d-\frac{d d^{\prime} T_{s}}{2 L} v_{o f f},
$$

of which satisfaction is equivalent to satisfaction of both equations in (2), simultaneously.

Equation (4) is a unified large-signal expression which describes how a duty ratio $d$ is determined. Small-signal information can be derived by its perturbation, which yields

$$
\begin{aligned}
\hat{i}_{l}= & \hat{i}_{c}-\left[M_{c}+\frac{\left(D^{\prime}-D\right) V_{o f f}}{2 L}\right] \\
& \cdot T_{s} \hat{d}-\frac{D D^{\prime} T_{s}}{2 L} \hat{v}_{o f f} .
\end{aligned}
$$

Then, the unified modulator model is obtained as

$$
\begin{aligned}
\hat{d}= & \frac{1}{\left[M_{c}+\left(D^{\prime}-D\right) V_{o f f} / 2 L\right] T_{s}} \\
& \cdot\left[\left(\hat{i}_{c}-\hat{i}_{l}\right)-\frac{D D^{\prime} T_{s}}{2 L}\left(\alpha \hat{v}_{g}+\beta \hat{v}\right)\right]
\end{aligned}
$$

where the expression $\hat{v}_{o f f}=\alpha \hat{v}_{g}+\beta \hat{v}$ has been used, with $\alpha=1,0$, and 1 , and $\beta=0,1$, and 1 , respectively, for the buck, the boost, and the buck-boost converters.

Hence, the following identifications can be made in Fig. 2 :

$$
\begin{aligned}
F_{m} & =\frac{1}{\left[M_{c}+\left(D^{\prime}-D\right) V_{o f f} / 2 L\right] T_{s}} \\
k & =\frac{D D^{\prime} T_{s}}{2 L} .
\end{aligned}
$$

The unified modulator model of (6) is a modification of that proposed in [5], and the modulator gain factor $F_{m}$ is equivalent to that of [12]. In terms of $F_{m}$ and $k$, (6) becomes

$$
\hat{d}=F_{m}\left[\left(\hat{i}_{c}-\hat{i}_{l}\right)-k\left(\alpha \hat{v}_{g}+\beta \hat{v}\right)\right]
$$

A useful parameter can be identified by rewriting (7) as

$$
F_{m}=\frac{2 L}{T_{s} V_{o f f}} \frac{1}{\left[\left(\frac{2 L M_{c}}{D^{\prime} V_{o f f}}+2\right) D^{\prime}-1\right]} .
$$

It reveals that $F_{m}$ approaches infinity for $D^{\prime}=$ $1 /\left[2 L M_{c} / D^{\prime} V_{o f f}+2\right]$, which is the minimum value for $D^{\prime}$ to maintain a finite positive value for $F_{m}$. Thus, it is useful to identify this particular value as

$$
D_{\min }^{\prime} \equiv \frac{0.5}{\left(1+\frac{M_{c}}{D^{\prime} V_{o f f} / L}\right)} \equiv \frac{0.5}{\left(1+\frac{M_{c}}{M_{1}}\right)} .
$$

It is obvious that $M_{c}$, normalized with respect to a certain value, extends stability for $D_{\min }^{\prime}<0.5$, or $D_{\max }=1-$ $D_{\min }^{\prime}>0.5$, i.e., $D_{\min }^{\prime}$ exhibits the stabilizing effect of $M_{c}$ explicitly (more on this issue later).

Moreover, the unified modulator model indicates that a necessary condition for the current loop to be stable is that $F_{m}$ remains positively finite, which in turn ensures that the current feedback remains negative and finite. The condition for $F_{m}$ to be positively finite is simply

$$
D^{\prime}>D_{\min }^{\prime}
$$

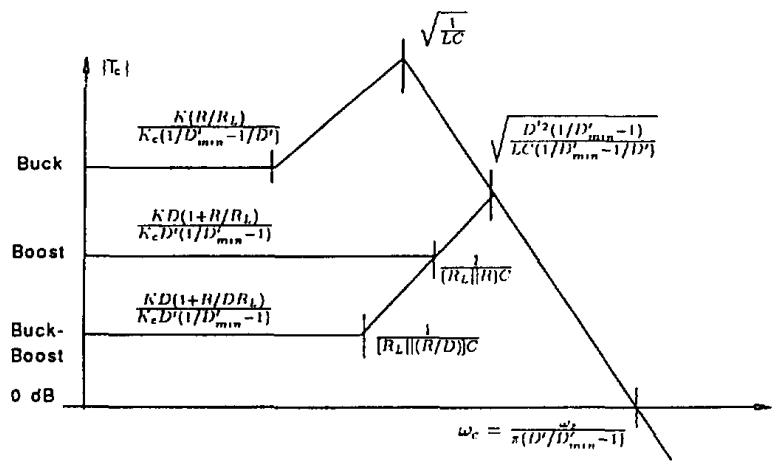

Fig. 5. Current-loop gains for three basic converters. The extrapolated crossover frequency $\omega_{c}$ approaches infinity if $D^{\prime} \rightarrow D_{\text {min }}^{\prime}$, the stability limit. Expressions for $T_{C}(0)$ reveal that the mechanism for instability for the buck is different from those for the boost and the buck/boost converters.

which is exactly the conventional condition for stability of the current loop detailed in [5].

In terms of $D_{\min }^{\prime}$, a low-entropy expression [22] for $F_{m}$ is obtained as

$$
F_{m}=\frac{2 L}{T_{s} V_{o f f}} \frac{1}{\left(D^{\prime} / D_{\min }^{\prime}-1\right)} .
$$

Several physical interpretations are immediately available from low-entropy expressions for $F_{m}$ and $k$ in (9). The first factor in the right of (13) is a measure for the value of slopes since (3) shows that both $M_{1}$ and $M_{2}$ are proportional to the ratio $V_{\text {off }} / L$. The second factor represents the effect that the compensating ramp $M_{c}$ has on the gain factor. The value of $k$ in (8) reflects the effect that perturbations of slopes $M_{1}$ and $M_{2}$ have on duty ratio. The larger the inductance $L$ is, the smaller the effect is, since both slopes are in reciprocal proportion to inductance. The product $D D^{\prime}$ reveals that at both extremes of the duty ratio, the effect due to slope perturbations is vanishingly small.

Also, a geometric interpretation of the proposed unified modulator model (an original version appeared in [21]) is found in [7]. The derivation in [7] was based on the averaged current in perturbed state, which is similar to [6], but with unequal values of the current at the start and the end of each switching cycle.

\section{CURRENT-LoOP GAIN}

This section derives expressions for current-loop gain for three basic converters and discusses their salient features.

\section{A. Current-Loop Gain}

Current-loop gain is derived by following the steps in [5]. The results, shown in Fig 5, are qualitatively the same as those in [5]. The most salient feature is that the final asymptote is the same for all three converters, crossing zero $\mathrm{dB}$ at $\omega_{c}$. This property reflects the fact that at high-frequencies, converter dynamics is determined by switches and inductances only. The expression for $\omega_{c}$ is of the form

$$
\omega_{c}=\frac{\omega_{s}}{\pi\left(D^{\prime} / D_{\min }^{\prime}-1\right)}
$$


which is in terms of the operating point parameter $D^{\prime}$ relative to its minimum value $D_{\min }^{\prime}$ for stability, and $\omega_{c}$ approaches infinity when $D^{\prime}$ approaches $D_{\min }^{\prime}$, i.e., in the stability limit.

Extension of $T_{c}(s)$ to include the sampling effect shows that $\omega_{c}$ is not necessarily the current loop crossover frequency because of the presence of an additional pole in $T_{c}(s)$. For this reason, $\omega_{c}$ will from now on be called the extrapolated crossover frequency of current-loop gain.

It is interesting to note that expressions for $T_{c}(0)$ can be shown to be identical to those presented in [5], where a different value for the modulator gain $F_{m}$ was used. This surprising result seems to suggest that the dc value of a current-loop gain is independent of the value of the modulator gain. Indeed, it can be shown that this is generally true [23]. Expressions for current-loop gains reveal that there are two different mechanisms for a current loop to become unstable. For a buck converter, the double pole is fixed in position. The extrapolated crossover frequency $\omega_{c}$ approaches infinity when $T_{c}(0)$ becomes unlimited. Note that in reality, $T_{c}(0)$ will be limited by parasitic resistance that inevitably exists in a converter. For a boost or a buck/boost, the double pole changes in position with different values of the compensating ramp and the duty ratio. $T_{c}(0)$ is always finite. In these two cases, $\omega_{c}$ goes to infinity together with the double pole in the stability limit.

The values $\omega_{c}$ and $T_{c}(0)$ can be effectively controlled by introducing different values for the compensating ramp, if duty ratio and inductance are kept the same. The larger the ramp slope is, the lower the values of $\omega_{c}$ and dc gain are. A rule of thumb may be set as that for $M_{c}=M_{2}, \omega_{c} \approx \omega_{s} / 3$. In an actual design, it may be necessary to use a value of $M_{c}$ larger than $M_{2}$ to accommodate possible degradation caused by possible parasitic reactances associated with current sensing circuitry.

\section{SAMPLING EFFECT}

The term "sampling" is used here to refer to the fact that duty ratio is determined only once in a switching cycle. Two independent approaches yield the same result: Only one additional pole $\omega_{p}$ needs to be added to a current-loop gain derived from the low-frequency model to represent the sampling effect.

\section{A. Additional Pole $\omega_{p}$ : First Approach}

The first approach is to find a rational function approximation of the sampled representation of the closed-loop transfer function $\hat{i}_{l} / \hat{i}_{c}$ of the current-programmed power stage, from which the presence of one additional pole in $T_{c}(s)$ is inferred.

From the geometry of the currents in Fig. 4, one can write two discrete-time equations:

$$
\left\{\begin{array}{l}
\Delta i_{c, n+1}=\Delta i_{l, n}+M_{1} \Delta t_{n+1}+M_{c} \Delta t_{n+1} \\
\Delta i_{l, n+1}=\Delta i_{c, n+1}-M_{c} \Delta t_{n+1}+M_{2} \Delta t_{n+1}
\end{array}\right.
$$

Two results can be obtained from this set of equations: one is the duty-ratio modulator gain $F_{m}$, and the other is a discretetime transfer function $\Delta i_{l} / \Delta i_{c}$.
Information about the duty ratio can be obtained by solving for $\Delta t_{n+1}$ in (15). Subtraction of the first expression from the second and then rearranging yields

$$
\hat{d}_{n+1} \equiv \frac{\Delta t_{n+1}}{T_{s}}=\frac{\Delta i_{c, n+1}-\left(\Delta i_{l, n+1}+\Delta i_{l, n}\right) / 2}{\left[M_{c}+\left(M_{1}-M_{2}\right) / 2\right] T_{s}} .
$$

With the restriction of low-frequency perturbation, one has $\Delta i_{l, n+1} \approx \Delta i_{l, n}$, which in turn gives

$$
\begin{aligned}
\hat{d} & =\frac{\hat{i}_{c}-\hat{i}_{l}}{\left[M_{c}+\left(M_{1}-M_{2}\right) / 2\right] T_{s}} \\
& =\frac{2 L}{T_{s} V_{\text {off }}} \frac{1}{\left(D^{\prime} / D_{\min }^{\prime}\right)}
\end{aligned}
$$

which shows the same coefficient as in (6) and verifies the expression for the modulator gain $F_{m}$. The low-frequency restriction is justified for the derivation of $F_{m}$, since it is shown in [18] that no frequency dependency is associated with $F_{m}$.

In addition, the discrete transfer function from control $\Delta i_{c}$ to inductor current $\Delta i_{l}$ is derived by introduction of the $z$ transform into the two equations in (15), which leads to

$$
\frac{\Delta i_{l}(z)}{\Delta i_{c}(z)}=a \frac{z}{z-1+a}
$$

where $a \equiv\left(M_{1}+M_{2}\right) /\left(M_{1}+M_{c}\right)=2 D_{\min }^{\prime} / D^{\prime}$.

To derive a continuous expression for the current transfer function, two more relationships are needed: 1) the connection between the $z$ domain and the sampled-Laplace domain; and 2) the connection between the sampled- and the continuousLaplace domains.

The first is the identity $z=e^{s T_{s}}$. The second is provided by the concept of "equivalent hold" proposed in [16], which states: In the small-signal limit, the continuous quantity $\hat{i}_{l}$ is related to its sampled counterpart by the transfer function of a zeroth-order-hold circuit.

Applying this property, one obtains

$$
\hat{i}_{l}(s)=a \frac{e^{s T_{s}}}{e^{s T_{s}}-1+a} \frac{1-e^{-s T_{s}}}{s} \hat{i}_{c}^{*}(s)
$$

where the asterisk represents a sampled quantity and

$$
\begin{aligned}
\hat{i}_{c}^{*}(s) & =\frac{1}{T_{s}} \sum_{n=-\infty}^{\infty} \hat{i}_{c}\left(s+\frac{j 2 n \pi}{T_{s}}\right) \\
& \approx \frac{1}{T_{s}} \hat{i}_{c}(s) .
\end{aligned}
$$

The approximation is made possible by the fact that the control current can be taken to be a pure sinusoid, and hence contributions of sidebands are negligible if a narrow-band analyzer is used for measurement.

To simplify further the current transfer function, one can invoke the following modified Pade approximation for the complex exponential, originated implicitly in [8]:

$$
e^{-s T_{s}}=\frac{1-\frac{1}{2 / \pi}\left(\frac{s}{\omega_{s} / 2}\right)+\left(\frac{s}{\omega_{s} / 2}\right)^{2}}{1+\frac{1}{2 / \pi}\left(\frac{s}{\omega_{s} / 2}\right)+\left(\frac{s}{\omega_{s} / 2}\right)^{2}}
$$


which enjoys good accuracy up to half the switching frequency. Substitution of (21) into (19) yields

$$
\frac{\hat{i}_{l}(s)}{\hat{i}_{c}(s)}=\frac{1}{1+\frac{1}{Q_{s}}\left(\frac{s}{\omega_{s} / 2}\right)+\left(\frac{s}{\omega_{s} / 2}\right)^{2}}
$$

where $Q_{\mathrm{s}}=2 /[\pi(2 / a-1)]$, or

$$
Q_{s} \equiv \frac{2}{\pi\left(D^{\prime} / D_{\min }^{\prime}-1\right)} .
$$

It is seen that the current transfer function is a standard lowpass quadratic with its corner located at half the switching frequency, and whose Q-factor $Q_{s}$ can be effectively controlled by the compensating ramp $M_{c}$ through the parameter $D_{\min }^{\prime}$.

Moreover, the expression for $Q_{s}$ reveals explicitly that oscillation is possible if $Q_{s}$ goes to infinity, that is, if the inequality $D^{\prime}>D_{\min }^{\prime}$ is violated, which is exactly the conventional criterion for stability of the current loop [3], [5]. Since this potential oscillation will be at half the switching frequency, it is commonly referred to as subharmonic oscillation.

The denominator of (22) is typical of the closed-loop response of a system having a two-pole loop gain in the neighborhood of the crossover frequency. If in this neighborhood the loop gain is $T_{c}^{\prime}(s)$, then $T_{c}^{\prime}(s)$ can be inferred from

$$
\frac{T_{c}^{\prime}}{1+T_{c}^{\prime}}=\frac{1}{1+\frac{1}{Q_{s}}\left(\frac{s}{\omega_{s} / 2}\right)+\left(\frac{s}{\omega_{s} / 2}\right)^{2}}
$$

which leads to

$$
T_{c}^{\prime}(s)=\frac{1}{\frac{s}{\omega_{c}}\left(1+\frac{s}{\omega_{p}}\right)}
$$

where

$$
\begin{aligned}
& \omega_{p}=\frac{\left(\omega_{s} / 2\right)}{Q_{s}}=\frac{\pi \omega_{s}\left(D^{\prime} / D_{\min }^{\prime}-1\right)}{4} \\
& \omega_{c}=\left(\omega_{s} / 2\right) Q_{s}=\frac{\omega_{s}}{\pi\left(D^{\prime} / D_{\min }^{\prime}-1\right)} .
\end{aligned}
$$

It is seen that $\omega_{c}$ is identical to that shown in Fig. 5 which identifies the high-frequency asymptote of $T_{c}(s)$, and hence the sampling effect can be considered responsible for introducing an additional pole $\omega_{p}$ in $T_{c}(s)$. It can therefore be concluded that the current-loop gains $T_{c}$ of Fig. 5 may be augmented by the additional pole $\omega_{p}$ to account for the sampling effect.

\section{B. Additional Pole $\omega_{p}$ : Second Approach}

The second approach gives straightforward physical insight into the problem of how to include the sampling effect in current loop. It represents a further development of and a significant departure from its original form presented in [4].

Figure 6 shows a sequence of events for deriving a highfrequency correction factor for current-loop gain. Fig. 6(a) is a block-diagram representation of a current-programmed converter for the evaluation of current-loop gain. Current-loop

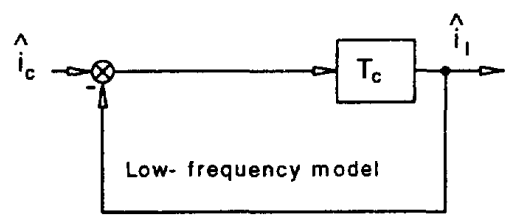

(a)

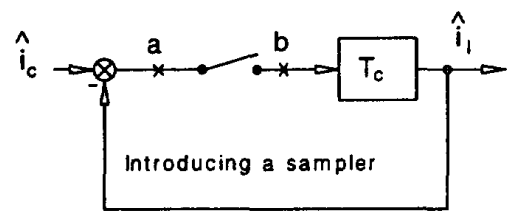

(b)

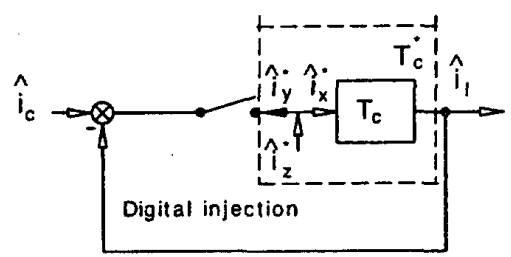

(c)

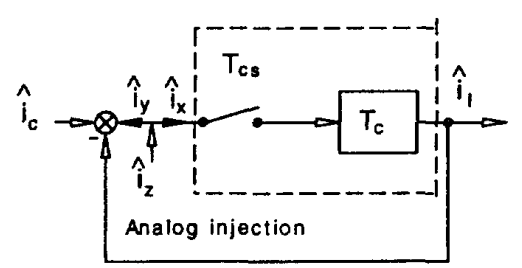

(d)

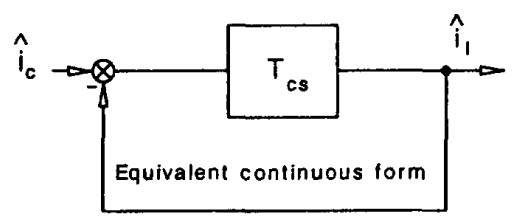

(e)

Fig. 6. Derivation of the additional pole. (a) A low-frequency current-loop gain. (b) A practical sampler is introduced to account for the sampling effect. (c) A digital injection measures a sampled current-loop gain $T_{c}^{*} \equiv \hat{i}_{y}^{*} / \hat{i}_{x}^{*}$. (d) An analog injection measures a hybrid current-loop gain $T_{c s} \equiv \hat{i}_{y} / \hat{i}_{x}$ and $T_{r s}$. (e) Absorbs the sampler to give an equivalent continuous form.

gain $T_{c}$ is the one derived from a low-frequency model such as the unified low-frequency model.

To represent the sampling mechanism, a practical sampler can be introduced immediately in front of the duty-ratio modulator as shown in Fig. 6(b). The term "practical" is used here to imply two things: 1) the sampling is done by a series of pulses, not impulses; 2) the sampling is quasiperiodic. The former will have profound impact on the evaluation of sampled-data current-loop gain. The latter has, as shown before in the previous section, negligible consequence. 
Because of the presence of the sampler, current-loop gain is not uniquely defined.

Injection at point "b" in Fig. 6 identifies an current-loop gain corresponding to digital injection.

Injection at point "a," shown in Fig. 6(d), defines a currentloop gain which corresponds to analog signal injection. This is so because the difference of two continuous signals, $\left(\hat{i}_{c}-\hat{i}_{l}\right)$, is still a continuous signal. An important feature of analog injection at point " $a$ " is that it is possible to absorb the sampler to yield an equivalent analog current-loop gain with the sampling effect accounted for. Indeed, if the big box in Fig. 6(d) is treated as a black box, the block diagram can be reduced to the one shown in Fig. 6(e) with continuous input and output. The resultant gain is a "new" current-loop gain $T_{c s}(s)$, where subscript " $s$ " indicates the fact that the sampling effect has been accounted for. The next step is the evaluation of the new current-loop gain $T_{c s}(s)$. Fig. 6(d) shows an analog injection measurement, from which one can derive

$$
T_{c s}(s)=T_{c}(s)\left[\frac{1}{1+T_{c}^{*}(s)-T_{c}(s)}\right] .
$$

That is, $T_{c s}(s)$ is simply $T_{c}(s)$, the current-loop gain obtained from the low-frequency model, multiplied by a correction factor $1 /\left[1+T_{c}^{*}(s)-T_{c}(s)\right]$.

It needs to be pointed out that one can find the original form of (28) in [4]. What is different here is that the evaluation of $T_{c}^{*}(s)$ will have to depart from the original approach as elaborated below.

In-depth discussion of sampled-data modeling in [4] collected three equivalent ways to evaluate $T_{c}^{*}(s)$ for a given $T_{c}(s)$. Here, only one approach will be discussed for brevity. For a given Laplace spectrum, say $T_{c}(s)$, the corresponding sampled-data spectrum $T_{c}^{*}(s)$ is given by

$$
T_{c}^{*}(s)=\frac{1}{T_{s}} \sum_{n=-\infty}^{\infty} T_{c}\left(s+\frac{j 2 n \pi}{T_{s}}\right)
$$

which is one particular form of the well-known Shannon's sampling theorem [19], showing explicitly the well-known phenomenon of frequency folding and aliasing.

Unfortunately, this formula cannot be applied directly to the evaluation of the sampled current-loop gain. The reason is that in Shannon's theorem, the sampling process is modeled by a series of impulses. For a physical switching converter, this constraint is too strong to be true.

Two things need to be discussed. First, in a switching converter, duty-ratio- or current-programmed, a value of duty ratio is determined when the ramp reaches the peak value set by the control signal. The control is actually exercised in a brief, but finite moment, once in a switching cycle, since a small, but finite time duration is required for the modulator to respond. To accomplish it instantaneously, an ideal modulator, i.e., a modulator with instantaneous response time, is needed.

Another aspect is that, in a measurement, duty ratio perturbation is still finite, even if a duty ratio is assumed to be determined instantaneously. This is so because in a measurement at least a perturbation of a few percent has to be provided in order to maintain a certain signal-to-noise ratio at the input port of the analyzer.
Evaluation of the sampled current-loop gain is pursued by working with geometries of current waveforms to derive discrete expressions. From the geometries of current waveforms in Fig. 4, (16) is obtained. Introducing z-transform into it leads to

$$
\hat{d}^{*}(z)=-\frac{1}{2}\left(1+z^{-1}\right) F_{m} \hat{i}_{l}^{*}(z)
$$

which, upon substitution of $z=e^{s T_{s}}$ and use of the concept of equivalent hold, yields the following expression for the sampled current-loop gain,

$$
\hat{d}^{*}(s)=-\frac{1}{2}\left(1+e^{-s T_{s}}\right) F_{m}\left(\frac{s}{1-e^{s T_{s}}}\right) \hat{i}_{l}(s) .
$$

Substitution of the modified Padé approximation and simplification leads to

$$
\hat{d}^{*}(s)=-F_{m}\left[1+\left(\frac{s}{\omega_{s} / 2}\right)^{2}\right] \frac{1}{T_{s}} \hat{i}_{l}(s) .
$$

Refer to Fig. 6(c). The sampled current-loop gain can be written as

$$
\begin{aligned}
T_{c}^{*}(s) & =\frac{\hat{d}_{y}^{*}(s)}{\hat{d}_{x}^{*}(s)}=-\frac{\hat{d}^{*}(s)}{\hat{\hat{i}}_{l}(s)} \cdot \frac{\hat{i}_{l}(s)}{\hat{d}_{x}^{*}(s)} \\
& =\left[1+\left(\frac{s}{\omega_{s} / 2}\right)^{2}\right] T_{c}(s) .
\end{aligned}
$$

Since $T_{c}^{*}$ differs from $T_{c}$ only at the high frequencies, the high-frequency asymptote is sufficient for the expression for $T_{c}$, i.e,

$$
T_{c}=\frac{\omega_{c}}{s} \text {. }
$$

Therefore, the current-loop gain with the sampling effect accounted for is found to be given by

$$
\begin{aligned}
T_{c s}(s) & =\frac{T_{c}(s)}{1+T_{c}^{*}(s)-T_{c}(s)} \\
& =\frac{T_{c}(s)}{1+T_{c}(s)\left[1+\left(\frac{s}{\omega_{c} / 2}\right)^{2}-1\right]} \\
& =\frac{T_{c}(s)}{\left(1+\frac{s}{\omega_{p}}\right)}
\end{aligned}
$$

where $\omega_{p}=\left(\omega_{s} / 2\right)^{2} / \omega_{c}=\pi \omega_{s}\left(D_{\min }^{\prime} / D-1\right) / 4$, which is identical to the value derived in (26). Hence, it is established again that accounting for the sampling effect introduces an additional pole $\omega_{p}$ into the current-loop gain.

\section{C. "Stability Parameter" $Q_{s}$ and High-Frequency Dynamics}

Having identified the additional pole, its influences on the high-frequency dynamics of the current-loop gain can be characterized.

The most striking feature of the high-frequency dynamics is that the high-frequency magnitude asymptote is fixed both in its slope and in its position. The asymptote is a straight line with a $-40 \mathrm{~dB} / \mathrm{dec}$ slope and crosses over $0 \mathrm{~dB}$ at half 


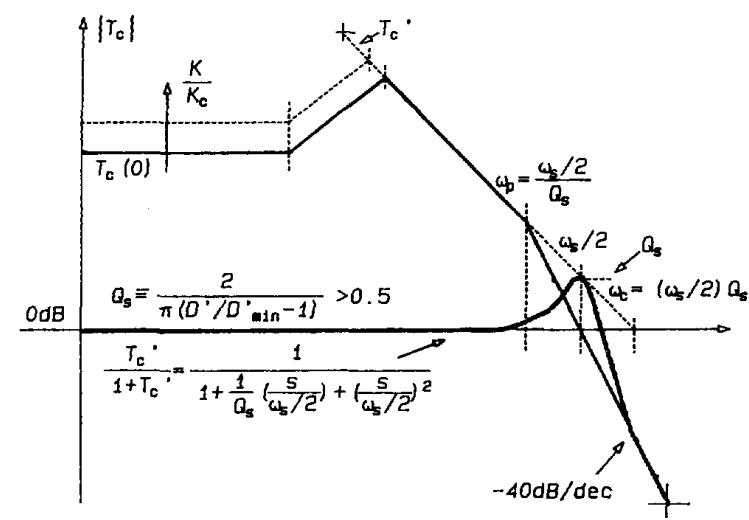

Fig. 7. Current-loop gain for "stability parameter" $Q_{s}>0.5 . T_{c}$ c crosses over at $\omega_{s} / 2$ on a double slope; closed-loop quadratic peaks up.

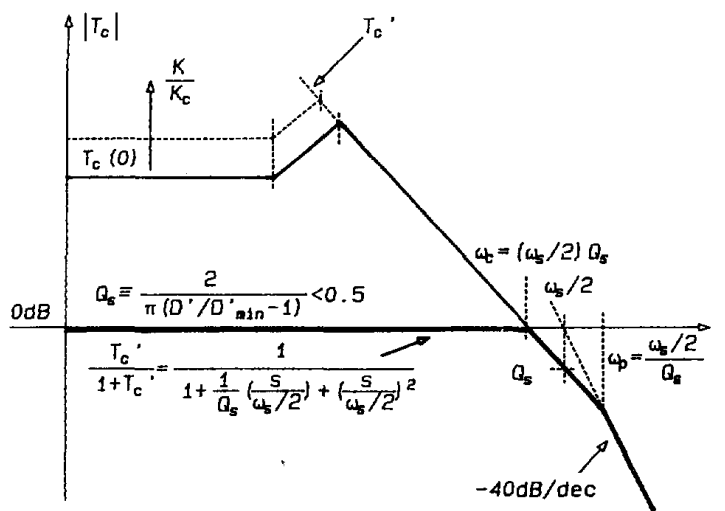

Fig. 8. Current-loop gain for $Q_{s}<0.5 . T_{r}$ crosses over at $\omega_{c}$ on a single slope; closed-loop quadratic has real poles at $\omega_{c}$ and $\omega_{p}$.

the switching frequency $\omega_{s} / 2$. Choice of different values of parameters will only move the low-frequency asymptotes up or down all together. The shape of them remain unchanged. Even though the extrapolated crossover frequency $\omega_{c}$ can approach infinity, the additional pole $\omega_{p}$ will provide sufficient attenuation at high frequencies which limits the actual crossover to half the switching frequency.

High-frequency dynamics are directly determined by the value of $Q_{s}$. Two cases of interest, for two values of $Q_{s}$, are shown in Figs. 7 and 8. In each, appropriate expressions for $T_{c}(0)$ and adjacent corner frequencies can be taken from Fig. 5 for the different converters. Increasing $K / K_{c}$ (larger inductance) increases only the low-frequency range of $T_{c}(s)$.

In Fig. 7 for $Q_{s}>0.5, T_{e}$ crosses over at $\omega_{s} / 2$ on a double slope and the resulting closed-loop response, represented by $T_{c}^{\prime} /\left(1+T_{c}^{\prime}\right)$, has a corresponding peak at $\omega_{s} / 2$.

In Fig. 8, for $Q_{s}<0.5, T_{c}$ crosses over at $\omega_{c}$ less than $\omega_{s} / 2$ on a single slope, and the additional pole $\omega_{p}$ is beyond crossover. The resulting closed-loop response has two real poles $\omega_{c}$ and $\omega_{p}$.

A wealth of useful design-oriented information is available from these low-entropy results. First, the final $-40 \mathrm{~dB} / \mathrm{dec}$ asymptote for $T_{c}$ is fixed in position, crossing $0 \mathrm{~dB}$ at $\omega_{s} / 2$.
Whether current-loop gain $T_{c}$ crossover occurs at or below $\omega_{s} / 2$ depends solely on $Q_{s}$, which is a function of the operating point $D^{\prime}$ relative to $D_{\min }^{\prime}$ given by (23). In turn, $D_{\min }^{\prime}$ is determined by the slope of the compensating ramp $M_{c}$, by (11).

Thus, if all quantities are considered constant except $M_{c}$, increasing $M_{c}$ results in a smaller $D_{\min }^{\prime}$ and a smaller $Q_{s}$, lowering $\omega_{c}$ and changing the configuration of $T_{c}$ from that of Fig. 7 toward that of Fig. 8. Alternatively, if all quantities are constant except the operating point parameter $D^{\prime}$, increasing $D^{\prime}$ (lower $D$ ) also results in a smaller $Q_{s}$ with the same consequences.

Since Figs. 7 and 8 illustrate the familiar loop-gain/closedloop relationships for a single-loop system, in which increasing loop-gain slope at crossover (lower phase margin) leads to peaking in the closed-loop response, it emerges that $Q_{s}$ is the central quantity of interest in the current-loop gain and could be referred to as a "stability parameter," since $Q_{s}$ approaches infinity in the stability limit of $D^{\prime}$ declining to $D_{\min }^{\prime}$. Further, a smaller $Q_{s}$ inexorably results in a lower $T_{c}(0)$.

Finally, a unified model for current-programmed converters with the sampling effect accounted for is obtained by expression of the modulator gain $F_{m}(s)$ in Fig. 2 as $F_{m} /\left(1+s / \omega_{p}\right)$. Note that the frequency dependence of $F_{m}$ is an "effective" representation of the sampling effect. A natural-sampled modulator alone does not have any frequency dependence [18].

\section{Measurement of Current-Loop Gain}

As discussed before, if a sampler is introduced into a model, different injection points define different loop gains. A problem tightly coupled with this ambiguity is measurement of current-loop gain. The uncertainty about proper ways of measuring current-loop gain and lack of thorough understanding of the issue have hindered progress in understanding of current programming, which may have been one of reasons for an unusually large number of different models competing with each other and, in some cases, with conflicting results.

\section{A. Digital Signal Injection Measurement}

Figure 9 illustrates one particular way of representing a digital injection measurement proposed in [17]. It injects a perturbation of duty ratio $\hat{d}_{z}^{*}$ at the output terminal of the duty ratio modulator. To represent its discrete nature, a sampler, shown ahead of the modulator, is introduced. From the simple model in Fig. 9, one can write:

$$
\hat{d}_{y}^{*}=\left[\hat{d}_{x}^{*} G_{d i}(s)\right]^{*} F_{m}=\left[G_{d i}(s) F_{m}\right]^{*} \hat{d}_{x}^{*}
$$

where $G_{d i}(s)$ denotes the duty-ratio-to-inductor-current transfer function, and $F_{m}$ denotes the modulator gain which has been taken to be a real number, although the actual values of these quantities are of no concern for this discussion. The above equation gives

$$
\frac{\hat{d}_{y}^{*}}{\hat{d}_{x}^{*}}=\left[G_{d i}(s) F_{m}\right]^{*}=T_{c}^{*}(s) .
$$

Therefore, the digital injection technique actually obtains a sampled version of current-loop gain $T_{c}(s)$. 


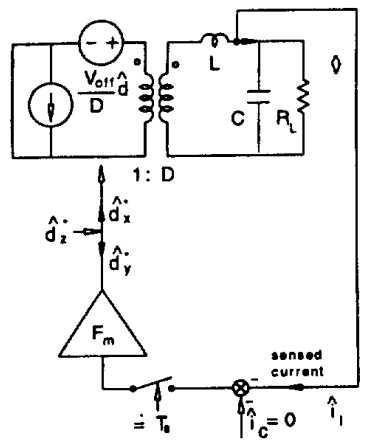

Fig. 9. Measurement of current-loop gain using digital injection $\hat{d}_{z}^{*}$ under the condition $\hat{v}_{g}=0$ and $\hat{i}_{c}=0$. The measured result $T_{c}^{*} \equiv \hat{d}_{y}^{*} / \hat{d}_{x}^{*}$ is a sampled version of the low-frequency current-loop gain $T_{c}$

If the sampler is ideal, sampled current-loop gain can be easily evaluated. As shown before, the high-frequency asymptote of current-loop gain can be expressed as $T_{c}^{\prime}(s)=$ $\omega_{c} / s$, whose sampled version is given by, according to [4],

$$
T_{c}^{*}(s)=\frac{\omega_{c} / f_{s}}{e^{s T_{s}}-1} .
$$

Substitution of the modified Padé approximation and simplification leads to

$$
T_{c}^{\prime *}(s)=\frac{\omega_{\mathrm{c}}}{s}\left[1-\frac{1}{2 / \pi}\left(\frac{s}{\omega_{s} / 2}\right)+\left(\frac{s}{\omega_{s} / 2}\right)^{2}\right] .
$$

Therefore, the overall sampled current-loop gain is obtained as

$$
T_{c}^{*}(s)=T_{c}(s)\left[1-\frac{1}{2 / \pi}\left(\frac{s}{\omega_{s} / 2}\right)+\left(\frac{s}{\omega_{s} / 2}\right)^{2}\right]
$$

which is exactly the form proposed in [8].

As pointed out in the discussion about sampling effect, in a switching converter, a sampler needs to be modeled as a practical sampler, i.e., a sampler which is quasiperiodic and has a finite sampling pulse width. This quasiperiodicity is characterized in [16], where it is shown that in the small-signal limit, the quasiperiodicity is inconsequential.

The finite sampling pulse width does have a consequence. It can be shown [23] that the fundamental component of the sampled version of a spectrum is given by

$$
G_{s}(j \omega) \approx \frac{1}{\pi} G(j \omega)
$$

Thus, the sampled spectrum will have a reduced amplitude. The attenuation is about one third, approximately $-10 \mathrm{~dB}$. This provides an explanation of the puzzling question: Why $\mathrm{dc}$ gains measured by digital injection are much lower than those obtained by analog injection measurement.

Another disadvantage associated with digital injection is that, according to (40), the sampled current-loop gain will always be folded over along half the switching frequency, regardless of the details of the loop gain around half the switching frequency. Hence, information about loop gain there and above will be lost.

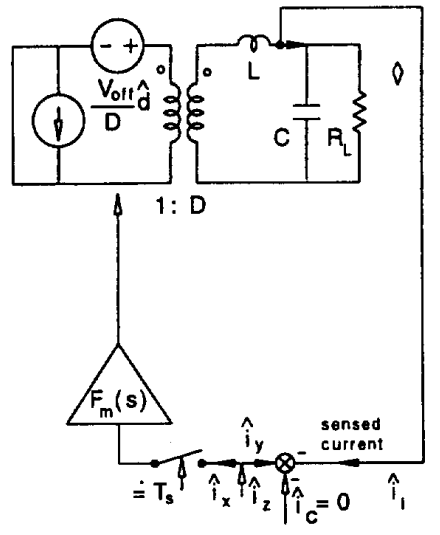

Fig. 10. Measurement of a current-loop gain by using analog injection $\hat{i}_{z}$ under the condition $\hat{v}_{g}=0$ and $\hat{i}_{c}=0$. The measured result $T_{c s} \equiv \hat{i}_{y} / \hat{i}_{x}$ is a hybrid version of $T_{c}$ and $T_{c}^{*}$. Whether a sampler is ideal or practical does not have any impact on the accuracy of an analog injection measurement.

\section{B. Analog Signal Injection Measurement}

An alternative can be found if one property of switching converters is fully appreciated. It can be easily seen that in a 2-switch converter, the transistor current is identical to the upgoing portion of the inductor current (in the case of multiple inductors, it will be the sum of all the inductor current). Hence sensing an inductor current is equivalent to sensing a transistor current. As a consequence, the sensed current is nonpulsating, which in turn suggests that a conventional analog signal injection technique [20] can be used.

Advantages of analog injection are almost exactly the disadvantages associated with digital injection technique. Analog signal injection is easy to use, with no need for special hardware. Since it injects and measures analog signals, no complications associated with sampling exist.

The only requirement is that the inductor current needs to be sensed, which is hardly a constraint at all for the following reason. For the purpose of measuring a current-loop gain, inductor current needs to be sensed. In practical implementations of designs, either transistor current or inductor current can be sensed (because of their equivalence), depending totally on practical considerations such as circuit reliability, cost, etc., as well as individual preferences.

In summary, analog injection can be used to measure current-loop gain if inductor current is sensed. For cases where both techniques are applicable, analog injection is the preferred technique since it outperforms digital injection in all aspects.

\section{EXPERIMENTAL VERIFICATIONS}

To verify theoretical predictions from the unified model and to check the proposed measurement technique, a prototype boost converter and a prototype buck converter have been constructed. Extensive measurements are performed. Analog signal injection is used to measure current-loop gain for the very first time. Good agreements between predictions and measurements are consistently seen for various different values of the duty ratio and the compensating ramp. 


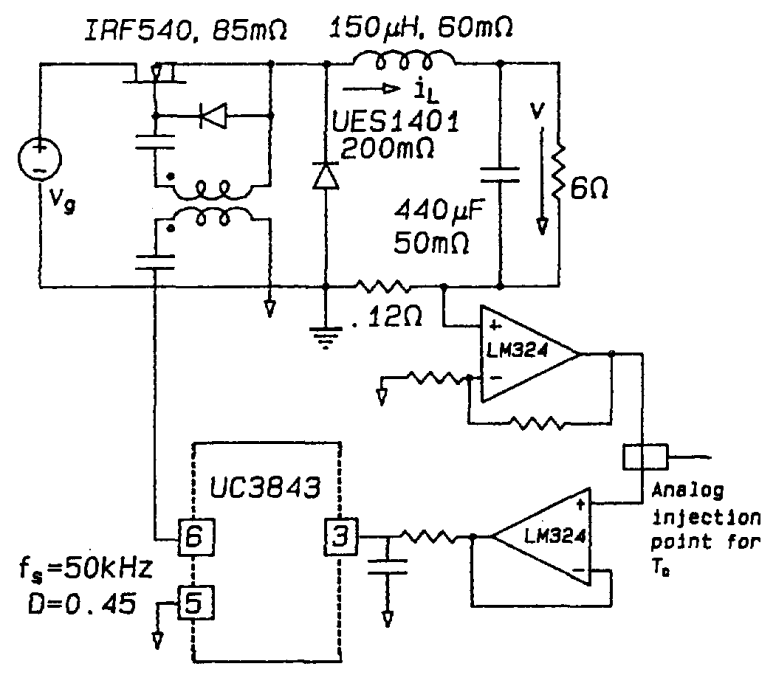

Fig. 11. Test buck converter.

Measured data for the buck converter are presented here with smooth curves of prediction to show accuracy of the proposed unified model.

\section{A. Measurements on a Buck Converter}

Figure 11 shows a test buck converter operating at 50 $\mathrm{kHz}$. The nonpulsating inductor current is sensed resistively. Two LM324s are used to reduce noise in the sensed inductor current, and to allow convenient analog signal injection.

Various values of compensating ramp slope $M_{c}$ are used to establish corresponding values of $D_{\min }^{\prime}$ and $Q_{s}$. All measurements are made at a converter operating point $D=0.45, D^{\prime}=$ 0.55 . A high value for duty ratio is intentionally chosen for the buck converter to test the accuracy of prediction of the unified model, since predictions are expected to degrade drastically as current-loop approaches its limit for stability. This is so because of one unique feature of a buck converter, which is that the dc gain $T_{c}(0)$, unlike the cases for a boost or a buck/boost, is expected to become unbounded in the stability limit. Hence, test under this situation is more demanding than lower values of duty ratio for the unified model.

Figure 12 shows the predicted smooth curves and measured results of current-loop gain for $D_{\min }^{\prime}=0.5\left(M_{c}=0\right)$. Since the operating point is $D^{\prime}=0.55$, little larger than $D_{\min }^{\prime}, Q_{s}$ is very high: from (23) $Q_{s}=6.4>1$, from (26) the additional pole is at $f_{p}=3.9 \mathrm{kHz}$, and the unified model predicts that $T_{c}$ crosses over at $f_{s} / 2=25 \mathrm{kHz}$ on a double slope. Agreement between prediction and measurement is good, and the presence of the additional pole $\omega_{p}$ can clearly be seen, especially from the phase measurement.

Figure 13 shows the same comparison for $D_{\min }^{\prime}=0.37$ $\left(M_{c}=M_{2} / 2\right)$. Since $Q_{s}=1.2>1, f_{p}=25 / 1.1=21 \mathrm{kHz}$ and $f_{c}=25 \times 1.2=29 \mathrm{kHz}$. Good agreement between two smooth curves is again seen in the entire frequency range of interest. The existence of $\omega_{p}$ is also seen in the amplitude and phase measurements.

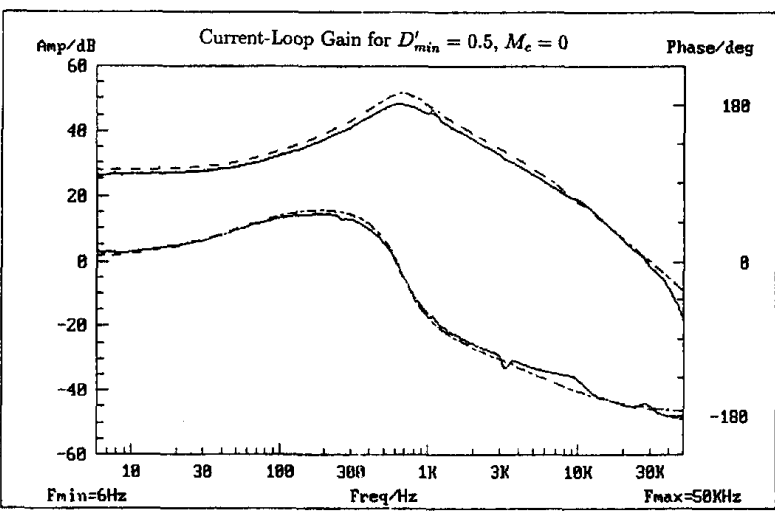

Fig. 12. Measured results (data points) and prediction (in asymptotes) of current-loop gain for a buck converter with $D_{\min }^{\prime}=0.50$. Good accuracy of the unified model predictions are clearly seen.

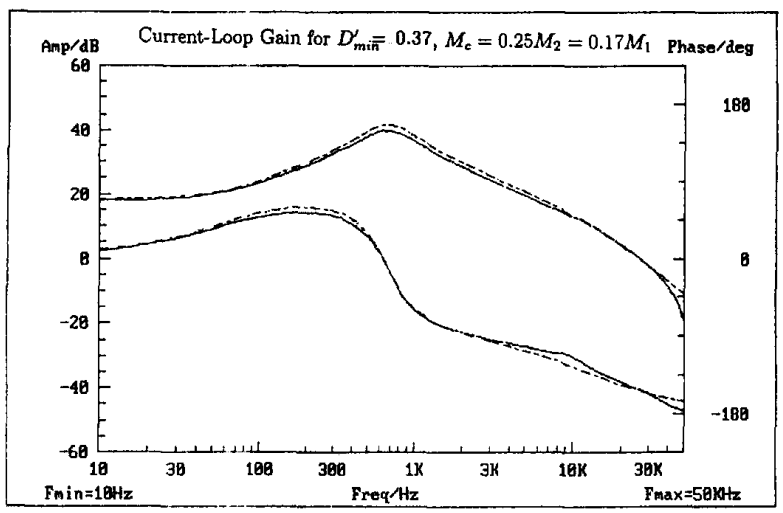

Fig. 13. Measured results (data points) and predicted asymptotes of current-loop gain for a buck converter with $D_{\min }^{\prime}=0.40$. Good accuracy of the unified model predictions are clearly seen.

Figure 14 shows again good agreement between the unified model prediction and the measured results of current-loop gain for $D_{\min }^{\prime}=0.3\left(M_{c}=M_{2}\right)$. Since $Q_{s}=0.64$ is now less than unity, $T_{c}$ crosses over on a single slope near $f_{c}=0.64 \times 25=16 \mathrm{kHz}$, showing that improved stability is obtained by increasing the compensating ramp to give a lower $Q_{s}$.

Figure 15 shows one more time good agreement between the unified model prediction and the measured data for the case where $D_{\min }^{\prime}=0.2\left(M_{c}=2 M_{2}\right)$. Since $Q_{s}=0.35$, much less than unity, $T_{c}$ crosses over on a single slope near $f_{c}=0.35 \times 25=8.8 \mathrm{kHz}$. It is seen that the effect of the additional pole is negligible, since it is located at $f_{p}=25 / 0.35=71 \mathrm{kHz}$, well above half the switching frequency. The current-loop for this case is much like a single pole system at high frequencies. Good agreement between two smooth curves is seen one more time.

Reviewing above comparisons, one concludes that the predictions for a buck converter by the unified model are verified. Note also that the double pole of the current-loop gain is shown to be fixed by the measured data for a buck converter. 


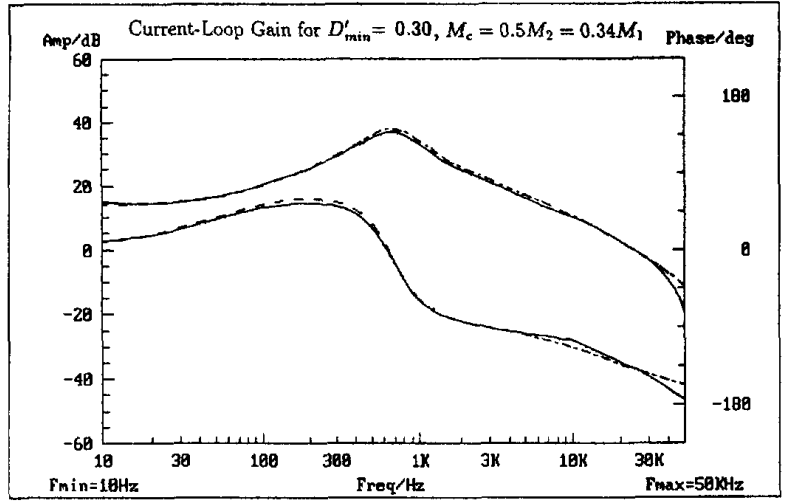

Fig. 14. Measured results (data points) and predicted asymptotes of current-loop gain for a buck converter with $D_{\min }^{\prime}=0.30$. The additional pole $\omega_{p}$ lies above $\omega_{s} / 2$. Good accuracy of the unified model predictions are clearly seen.

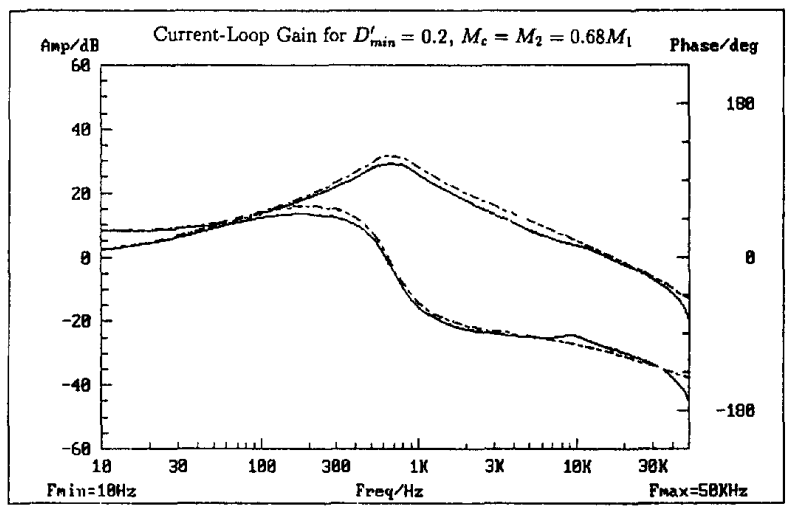

Fig. 15. Measured results (data points) and predicted asymptotes of current-loop gain for a buck converter with $D_{\min }^{\prime}=0.20$. The additional pole $\omega_{p}$ lies well above $\omega_{s} / 2$. Good accuracy of the unified model predictions are clearly seen.

Note that additional measured results on a boost prototype [21], [23] also support the unified model consistently.

It is therefore concluded that the unified model is theoretically sound and experimentally verified.

\section{CONCLUSION}

A unified model for a current-programmed converter is established as in Fig. 2. Although the format is the same as in [5], a modified modulator model leads to correspondingly modified expressions for $F_{m}$ and $k$ as given in (7) and (8), and also for the loop gain $T_{c}(s)$ shown in Fig. 5.

It is well-known that use of a compensating ramp of slope $M_{c}$ extends upwards from 0.5 the limit of the duty ratio $D$ for which stability is maintained. It is found convenient to introduce a new parameter $D_{\min }^{\prime}$, defined by $M_{c}$ as in (11), which represents the minimum value of the complementary duty ratio $D^{\prime}=1-D$ for which stability is maintained.

The sampling effect is here accounted for by an extension of the previous model from which the presence of an additional pole $\omega_{p}$ in $T_{c}(s)$, given by (26), is inferred and incorporated in the modulator gain $F_{m}(s)$ in Fig. 2, since they appear in cascade.

The presence of the additional pole $\omega_{p}$ causes the final high-frequency asymptote of $T_{c}(s)$ to be $-40 \mathrm{~dB} / \mathrm{dec}$, as shown in Figs. 7 and 8. A salient feature is that this final asymptote is fixed in position, crossing zero $\mathrm{dB}$ at $\omega_{s} / 2$. The loop-gain crossover frequency cannot exceed half the switching frequency regardless of any component values or converter operating point. Whether crossover occurs at or below $\omega_{s} / 2$ is determined by the value of the operating point $D^{\prime}$ relative to $D_{\min }^{\prime}$ through another parameter $Q_{s}$ defined in (23). The "stability parameter" $Q_{s}$ emerges as the central quantity of interest in the current-loop gain $T_{c}^{\prime}(s)$, since its value determines not only the crossover frequency but also the degree of peaking, which occurs at $\omega_{s} / 2$, in the low-pass quadratic of (22) that is contained in all of the closed-loop transfer functions.

It is revealed that inherent limitations exist for accuracy of a digital signal injection measurement [17]. The dc value of current-loop gain measured by digital injection is at least 10 $\mathrm{dB}$ below what it should be because of the finite pulse width of the practical sampler. To avoid complications caused by finite pulse width of the practical sampler, analog injection [20] can be used. It is found that analog injection outperforms digital injection in all aspects for the cases where both of them are applicable.

The unified model is verified by experimental measurements of current-loop gain on a buck and a boost converters. Agreements between predictions and measured data are consistently good. An analog signal injection technique is enabled by sensing the (nonpulsating) inductor current instead of the pulsating switch current, which does not change the nature of the current loop.

Figure 12 shows $T_{c}(s)$ for $Q_{s}=6.4$, for a buck. $Q_{s}$ has a high value since $D^{\prime}=0.55$ are little larger than $D_{\min }^{\prime}=0.5$; the presence of the additional pole $\omega_{p}$ at 3.9 $\mathrm{kHz}$, well below crossover, is clearly visible. The effect that the compensating ramp $M_{c}$ has on the current loop is clearly seen to be the stabilizing effect in Figs. 12-15. Current loop loses its stability when the corresponding current-loop gain loses its phase margin, an identical mechanism to that of a classical second-order low-pass system. Subtle differences in the way that current-programmed converters go to instability are also verified.

\section{REFERENCES}

[1] C. W. Deisch, "Simple switching control method changes power converter into a current source," IEEE PESC Record, pp. 300-306, 1978.

[2] A. Capel, G. Ferrante, D. O'Sullivan, and A. Weinberg, "Application of the injected current model for the dynamics of switching regulators with the new concept of LC3 modulator," IEEE PESC Record, pp. 135-147, 1978.

[3] S.-P. Hsu, A. Brown, L. Rensink, and R. D. Middlebrook, "Modeling and analysis of switching dc-to-dc converters in constant-frequency current-programmed mode," IEEE PESC Record pp. 284-301, 1979.

[4] A. B. Brown, "Topics in modeling, measurement, and design of high performance switching regulators," Ph.D. thesis, Power Electronics Group, California Institute of Technology, Pasedena, 1981.

[5] R. D. Middlebrook, "Topics in multiple-loop regulators and currentmode programming," IEEE PESC Record, pp. 716-732, 1985 (also in IEEE Trans. Power Electron., vol. 2, no. 2, pp. 109-124, 1987). 
[6] G. C. Verghese, C. A. Bruzos, and K. N. Mahabir, "Averaged and sampled-data models for current mode control: a re-examination," IEEE PESC Record, pp. 484-491, 1989.

[7] C. P. Shultz, "A unified model of constant frequency switching regulators using multiloop feedback control," in PCIM Proc., 1993, (subsection 6.3)pp. 319-329.

[8] R. B. Ridley, "A new, continuous-time model for current-mode control," IEEE Trans. Power Electron., vol. 6, no. 2, pp. 271-280, 1991.

[9] R. Tymerski and D. Li, "State-space models for currrent-programmed pulsewidth-modulated converters," IEEE Trans. Power Electron., vol. 8, no. 3, pp. 271-278, July 1993 .

[10] V. Vorpérian, "Analysis of current-mode controlled PWM converters using the model of the current-controlled PWM switch," in PCIM Conf. Record, 1990.

[11] R. D. Middlebrook and S. M. Cuk, "A general unified approach to modeling switching converter power stages," IEEE PESC Record, pp. $18-34,1976$

[12] F. C. Lee, Y. Yu, and M. F. Mahmoud, "A unified analysis and design procedure for a standardized control module for dc-to-dc switching regulators," IEEE PESC Record, pp. 284-301, 1980

[13] D. Maksimović and S. M. Cuk, "A unified analysis of PWM converters in discontinuous modes," in Int. PCIM Conf. Record, June 1989.

[14] S. Freeland and R. D. Middlebrook, "A unified analysis of converters with resonant switches," IEEE PESC Record, pp. 20-30, 1987.

[15] A. K. S. Bhat and F. D. Tan, "A unified approach to characterization of PWM and quasi-PWM converters: Topological constraints, classification, and synthesis," IEEE PESC Record, pp. 760-767, 1989 (also in IEEE Trans. Power Electron., vol. 6, no. 4, pp. 719-726, 1991).

[16] B. Y. B. Lau and R. D. Middlebrook, "Small-signal frequency response theory for piecewise-constant two-switched-network dc-to-dc converters," IEEE PESC Record, pp. 186-200, 1986.

[17] B. H. Cho and F. C. Lee, "Measurement of loop-gain with the digital modulator," IEEE PESC Record, pp. 363-373, 1984.

[18] R. D. Middlebrook, "Predicting modulator phase lag in PWM converter feedback loops," Powercon 8, Paper H-4, 1981.

[19] C. E. Shannon, "A mathematical theory of communication," Bell System Technical Journal, no. 3, vol. 27, pp. 379-433, July 1948.

[20] R. D. Middlebrook, "Measurement of loop gain in feedback systems," Int. J. Electron., vol. 38, no. 4, pp. 485-512.

[21] F. D. Tan and R. D. Middlebrook, "Unified modeling and measurement of current-programmed converters," IEEE PESC Record, pp. 380-387, 1993

[22] R. D. Middlebrook, "Low-entropy expressions: The key to designoriented analysis," in Proc. IEEE Frontiers in Education. Purdue University, West Lafayette, IN, 1991, pp. 399-403.

[23] F. D. Tan, "Modeling and control of switching converters: I. Unified modeling and measurement of currrent-programmed converters, II. A generic averaged model for switches in dc-to-dc converters," Ph.D. thesis, Power Electronics Group, California Institute of Technology, Pasadena, Nov, 1993.

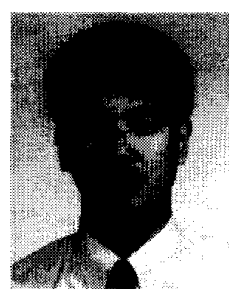

F. Dong Tan (M'95) received the B.E.Sc. degree with honors from the Jiangxi Polytechnic University, Nanchang, China, in 1979, and the Ph.D. degree from the California Institute of Technology, Pasadena, in 1993, both in electrical engineering.

From 1979 to 1986 , he was with the Faculty of Jiangxi Polytechnic University, where he taught several courses in circuits, electronics, and electric machines. From 1986 to 1987 , he was a Visiting Research Assistant Professor at the University of Waterloo, Ontario, Canada, where he applied bifurcation theory to switching converters. From 1987 to 1988 , he pursued graduate studies at the University of Victoria, British Columbia, Canada, where he worked on quasiresonant converters. In 1989, he was accepted directly into the Ph.D. program with the Power Electronics Group at Caltech, where he focused his research on modeling and control of $\mathrm{dc} / \mathrm{dc}$ converters and related magnetic design issues. Since November 1993, he has been a Senior Engineer with the QSC Audio Products, Inc., Costa Mesa, CA, developing soft-switched, high performance, and high power professional audio amplifiers.

Dr. Tan has published more than 10 peer-reviewed journal and conference papers. He also holds one U.S. patent and has several US and European patents pending. He received several awards for teaching excellence, presented by the Jiangxi Polytechnic University. He is currently serving as Vice Chairman/Program Chair of the Los Angeles Chapter of the IEEE Power Electronics Society.

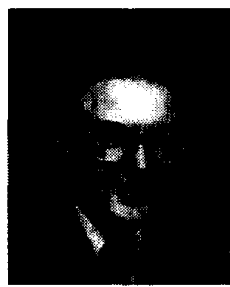

R. D. Middlebrook (LF'94) is Professor of Electrical Engineering at the California Institute of Technology, Pasadena.

His research interests, formerly in solid-state device modeling, are now in circuits and systems, and particularly in power processing electronics in which he is well-known as author and lecturer. He is especially interested in design-oriented circuit analysis and measurement techniques which he teaches at Caltech, and he has conducted short courses on his methods in both Europe and the United States. His publications include numerous papers, a book on solid-state device theory, and another on differential amplifiers.

Dr. Middlebrook received the 1982 IEEE William E. Newell Power Electronics Award for Outstanding Achievement in Power Electronics, a 1990 PCIM Award for Leadership in Power Electronics Education, and a 1982 Award for Excellence in Teaching, presented by the Board of Directors of the Associated Students of Caltech. In 1984, he received an IEEE Centennial Medal, and in 1991, he was awarded the Edward Longstreth Medal of the Franklin Institute. 